\title{
Computation of partial enthalpies of various Lennard- Jones model mixtures by NPT molecular dynamics
}

\author{
R Vogelsang $\dagger, C$ Hoheisel $\dagger$, P Sindzingre $\ddagger$, G Ciccotti $\ddagger$ and D Frenkel§ \\ † Theoretische Chemie, Fakultät Chemie, Ruhr-Universität Bochum, 4630 Bochum, \\ Federal Republic of Germany \\ $\ddagger$ Centre d'Etudes Nucleaires de Saclay, Section de Recherches de Metallurgie Physique, \\ 91191 Gif sur Yvette Cédex, France \\ $\S$ FOM Institute for Atomic and Molecular Physics, PO Box 41883, 1009 DB Amsterdam, \\ The Netherlands
}

Received 15 July 1988 , in final form 17 October 1988

\begin{abstract}
Exact calculation of the thermal diffusion coefficient, $D^{\mathrm{T}}$, of a binary mixture by molecular dynamics requires a separate determination of the partial enthalpies. A recently proposed method is used here to evaluate these quantities for Lennard-Jones binary liquid mixtures of which the component particles interact via very different pair potential functions. It is shown that the method works well for the 'non-ideal' mixtures considered. The 'exact' values of the partial enthalpies deviate significantly from those approximated by sharing the total enthalpy among the components proportionally to the composition. Thus the values of $D^{\mathrm{T}}$ recently computed for these mixtures have to be corrected appreciably, as expected.
\end{abstract}

\section{Introduction}

A reasonable definition of the heat current in a binary mixture requires the partial enthalpies [1-3]. Thus, computation of correlation functions for the thermal conductivity, $\lambda$, and the thermal diffusion coefficient, $D^{\mathrm{T}}$, by molecular dynamics (MD) methods involves an additional evaluation of the partial enthalpies, $h_{\alpha}(\alpha=1,2)$, of the twocomponent system. Recently, a Widom-like sampling method, the so-called difference method, has been proposed for the determination of 'exact' values of $h_{\alpha}$ [4]. It has been tested for the approximately 'ideal' Ar-Kr Lennard-Jones (LJ) mixture [5] and the results demonstrated the applicability of the method.

This difference method is used here for very 'non-ideal' binary liquid LJ mixture types. Type A consists of particles that differ substantially as regards the energy interaction parameter, while type B contains particles with very different volume parameters in the pair potential.

The accuracy of the computed data is discussed for six different mixtures at equimolar concentration as well as for the concentration dependence of one chosen mixture.

Finally, the correction factors for the transport coefficients of these systems, which have been calculated with the use of approximate partial enthalpies [6], are given. 


\section{The difference method}

As the difference method (DM) is presented in detail in $[4,5]$, we only outline it briefly here. The DM is a Widom-like method which samples the change in the potential energy that results from virtually interchanging a particle of a given species for a particle of another species. For a two-component system of species A and B, the DM directly provides the difference values between such excess quantities as the chemical potentials, the partial enthalpies, and the partial volumes. We concentrate here on the partial enthalpies, $h_{\mathrm{A}}$ and $h_{\mathrm{B}}$, which may be computed using the following formula:

$$
\begin{aligned}
\Delta h=h_{\mathrm{A}}-h_{\mathrm{B}} & =h_{\mathrm{A}}^{\mathrm{ex}}-h_{\mathrm{B}}^{\mathrm{ex}}=\left\langle\left(\Delta U^{\mathrm{A}+\mathrm{B}^{-}}+U\left(N_{\mathrm{A}}, N_{\mathrm{B}}\right)+P V\right)\right. \\
& \left.\times \exp \left(-\beta \Delta U^{\mathrm{A}+\mathrm{B}^{-}}\right)\right\rangle_{N_{\mathrm{A}}, N_{\mathrm{B}}} /\left\langle\exp \left(-\beta \Delta U^{\mathrm{A}+\mathrm{B}^{-}}\right\rangle_{N_{\mathrm{A}}, N_{\mathrm{B}}}\right.
\end{aligned}
$$

where $\Delta U^{\mathrm{A}+\mathrm{B}^{-}}$denotes the change in the potential energy resulting from the transformation of a particle $\mathrm{B}$ into a particle A-likewise $\Delta U^{\mathrm{B}+\mathrm{A}-}$ for a particle A. $U\left(N_{\mathrm{A}}, N_{\mathrm{B}}\right)$ denotes the potential energy of a binary mixture composed of $N_{\mathrm{A}}$ and $N_{\mathrm{B}}$ particles, where $N_{\mathrm{A}}+N_{\mathrm{B}}$ is the total number of particles of the system. $V$ denotes the volume, $P$ the pressure and $\beta=1 / k_{\mathrm{B}} T$ with $k_{\mathrm{B}}$ the Boltzmann constant and $T$ the temperature. The brackets indicate the ensemble average-here the NPT ensemble. Assuming pairwise interaction, we have for any particle $(i, \mathrm{~B})$

$$
\Delta U^{\mathrm{A}+\mathrm{B}-}=\sum_{\substack{\alpha=\mathrm{A}, \mathrm{B} \\(j, \alpha \neq i, \mathrm{~B})}} \sum_{j=1}^{N_{\alpha}}\left(\Phi_{\mathrm{A} \alpha}\left(r_{i j}^{\mathrm{B} \alpha}-\Phi_{\mathrm{B} \alpha}\left(r_{i j}^{\mathrm{B} \alpha}\right)\right)\right.
$$

where $\Phi_{\alpha \beta}(r)(\alpha, \beta=1,2 ; \alpha<\beta)$ denote the three pair potential functions occurring in a two-component system.

Denoting the total molar enthalpy of the mixture by $H$ and the mole fractions of the components by $x_{\mathrm{A}}$ and $x_{\mathrm{B}}$, the partial enthalpies $h_{\mathrm{A}}$ and $h_{\mathrm{B}}$ can be obtained via the following equations:

$H=N_{\mathrm{A}} h_{\mathrm{A}}+N_{\mathrm{B}} h_{\mathrm{B}} \quad h_{\mathrm{A}}=(1 / N) H+x_{\mathrm{B}} \Delta h \quad h_{\mathrm{B}}=(1 / N) H-x_{\mathrm{A}} \Delta h$.

Apparently, knowledge of $H$ and $\Delta h$ suffices for calculating the values for the partial enthalpies $h_{\alpha}$.

\section{Potentials, mixture systems and calculations}

Lennard-Jones (LJ) (12-6) potential functions have been used throughout. The potential parameters $\varepsilon_{\alpha \beta}$ and $\sigma_{\alpha \beta}$ were chosen by analogy with those of the model mixtures that have already been investigated in [6]. Two groups of mixture systems were studied. The first one contains systems composed of particles of very different interaction strength, the second one, systems composed of particles of very different 'volumes'. For both types of mixture, the unlike-potential interaction (A-B interaction) was varied systematically. We list the LJ potential parameters for the six mixtures considered in table 1.

Note that the notation of [6] has been used, and furthermore that the absolute values of the potential parameters have been chosen so that all the mixtures represent nearly the same one-fluid system at a reduced density of $n_{x}^{*}=0.75$ and a reduced temperature 
Table 1. Lennard-Jones potential parameters for the six model mixtures.

\begin{tabular}{|c|c|c|c|c|c|c|}
\hline System $\dagger$ & $\sigma_{\mathrm{AA}}(\AA)$ & $\sigma_{\mathrm{BB}}(\AA)$ & $\sigma_{\mathrm{AB}}(\AA)$ & $\varepsilon_{\mathrm{AA}} k_{\mathrm{B}}^{-1}(\mathrm{~K})$ & $\varepsilon_{\mathrm{BB}} k_{\mathrm{B}}^{-1}(\mathrm{~K})$ & $\varepsilon_{\mathrm{AB}} k_{\mathrm{B}}^{-1}(\mathrm{~K})$ \\
\hline A1) & \multirow{4}{*}{3.5375} & \multirow{4}{*}{3.5375} & \multirow{3}{*}{3.5375} & \multirow{3}{*}{100.0} & \multirow{3}{*}{200.0} & 120.0 \\
\hline A3 3 & & & & & & 150.0 \\
\hline A5 & & & & & & 180.0 \\
\hline B1 & & & {$[3.1838]$} & \multirow{3}{*}{141.45} & \multirow{3}{*}{141.45} & \multirow{3}{*}{141.45} \\
\hline B3 $\}$ & \multirow[t]{2}{*}{2.9479} & \multirow[t]{2}{*}{4.1271} & $3.5375\}$ & & & \\
\hline B5 & & & $(3.8913)$ & & & \\
\hline
\end{tabular}

$\dagger$ Notation from [6].

of $T_{x}^{*}=0.95$ [7]. By making this choice of LJ parameters we have ensured that the pressures of all the systems are approximately equal.

The MD calculations were performed in the NPT ensemble, following the formalism of Nosé-Andersen [4,5]. Details of these calculations may be found in [5]. Some important technical details of our runs are presented in table 2.

\section{Results}

\section{1. $A r-K r$}

To check our vector version of the MD programme, we reproduced all the Lennard-Jones $\mathrm{Ar}-\mathrm{Kr}$ data computed in [5]. While we achieved excellent agreement for all the quantities in general, some care was needed for states of low Ar mole fractions since the state point chosen in [5] is very close to the melting line of $\mathrm{Kr}$. To avoid the occurrence of metastable states during the MD computation, these latter runs were performed with 256 particles and equilibration was performed in the $N V E p$ ensemble, where $E$ denotes the total energy and $\boldsymbol{p}$ the total momentum of the system.

\subsection{Difference values of the chemical potential, partial volume, and partial enthalpy for system $A 1$ at different concentrations}

For system A1, which is a non-ideal one with a strong demixing tendency [8], we have calculated the difference quantities for the whole concentration range. Table 3 contains the difference values for the chemical potential, $\Delta \mu^{\mathrm{ex}}=\mu_{\mathrm{A}}^{\mathrm{ex}}-\mu_{\mathrm{B}}^{\mathrm{ex}}$, the partial volume $\Delta v=v_{\mathrm{A}}-v_{\mathrm{B}}$, and the potential enthalpy, $\Delta h=h_{\mathrm{A}}-h_{\mathrm{B}}$, as functions of the mole fraction of component $\mathrm{A}$. The statistical error for these quantities is about $3 \%$ in general.

Table 2. Technical details of the MD computations.

\begin{tabular}{ll}
\hline Particle number, $N$ & $108-256$ \\
Ensemble & $N P T(P$ : pressure; $T$ : temperature $)$ \\
Number of integration time steps & $10^{5}$ \\
Time step & $10^{-14} \mathrm{~s}$ \\
Integration scheme & Fourth-order Gear algorithm \\
Cut-off radii of the L potentials & $(2.5-3.3) \sigma_{\mathrm{AB}}$ \\
Computation time for 1000 steps on the Cyber 205 & $9.2 \mathrm{~s}(N=108)$ \\
& $36 \mathrm{~s}(N=256)$ \\
\hline
\end{tabular}


Table 3. Computed difference values for the chemical potential, the partial enthalpy, and the partial volume of system $\mathrm{A} 1\left(n^{*}=N \sigma_{\mathrm{AA}}^{3}=0.75 ; T^{*}=T k_{\mathrm{B}} / \varepsilon_{\mathrm{AA}}=1.28\right)$.

\begin{tabular}{llll}
\hline & $\begin{array}{l}\Delta \mu^{* \mathrm{ex}} \\
x_{\mathrm{A}}\end{array}$ & $\begin{array}{l}\Delta h^{*} \\
\left(\Delta h / N \varepsilon_{\mathrm{AA}}\right)\end{array}$ & $\begin{array}{l}\Delta v^{*} \\
\left(\Delta v / N \varepsilon_{\mathrm{AA}}\right)\end{array}$ \\
\hline 0.148 & 8.47 & 12.23 & 0.055 \\
0.25 & 7.62 & 11.18 & 0.195 \\
0.398 & 6.44 & 9.79 & 0.331 \\
0.5 & 5.65 & 8.99 & 0.365 \\
0.602 & 4.87 & 7.86 & 0.356 \\
0.75 & 3.77 & 6.39 & 0.348 \\
0.852 & 3.00 & 5.09 & 0.315 \\
\hline
\end{tabular}

In figure 1 we compare the values of $\Delta \mu^{* e x}$ for mixture $\mathrm{A} 1$ and $\mathrm{Ar}-\mathrm{Kr}$ as functions of the molar fraction. Mixture A1 shows a strong dependence of $\Delta \mu^{* \mathrm{ex}}$ on the composition, while $\Delta \mu^{* \mathrm{ex}}$ for $\mathrm{Ar}-\mathrm{Kr}$ remains nearly constant over the whole range of concentration. This behaviour of $\Delta \mu^{* \mathrm{ex}}$ for system $\mathrm{A} 1$ indicates a strong positive deviation of the Gibbs free energy from ideality. It corroborates our finding in [8] that an unsymmetric choice of the unlike-potential parameter generates non-ideality of a mixture.

\subsection{Partial enthalpies and correction factors for the thermal diffusion coefficients of systems of type $A$ and $B$}

While for the A systems the DM method works excellently with transformations of particles of both species, for B systems solely particles with the larger 'volume' are allowed to be transformed into those of smaller 'volume', for plausible reasons. Hence for the latter systems we doubled the number of runs to achieve results with an accuracy comparable to that achieved for the systems $\mathrm{A}$. The results for all the systems are summarised in table 4, which contains additionally the partial enthalpy differences calculated by distributing the total enthalpy among the component particles pro-

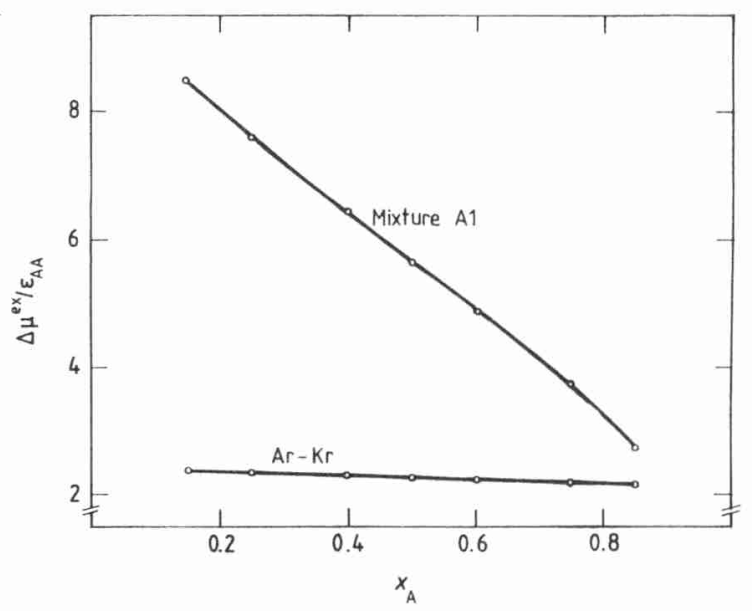

Figure 1. The excess chemical potential difference of system $\mathrm{A} 1$ and $\mathrm{Ar}-\mathrm{Kr}$ as a function of the mole fraction. $T^{*}=1.28 ; n^{*}=0.75$. 
Table 4. 'Exact' and approximated partial enthalpies (all the values without the potentialtail correction) calculated for the systems characterised in table 1 and $\mathrm{Ar}-\mathrm{Kr}$. Details for the systems (equi-molar composition): $T^{*}=(\mathrm{Al}) 1.28$, (A3) 1.43, (A5) 1.57; (B1) 0.95, (B3) 0.95, (B5) 0.95, (Ar-Kr) 0.98; $n^{*} \simeq$ (A1) 0.75, (A3) 0.75, (A5) 0.75; (B1) 0.75, (B3) 0.75, (B5) $0.75,(\mathrm{Ar}-\mathrm{Kr}) 0.75$.

\begin{tabular}{lllllll}
\hline \multirow{2}{*}{ System } & $\frac{h_{\mathrm{A}}^{* \text { mech }}}{T^{*}}$ & $\frac{h_{\mathrm{B}}^{* \text { mech }}}{T^{*}}$ & $\frac{h_{\mathrm{A}}^{*}}{T^{*}}$ & $\frac{h_{\mathrm{B}}^{*}}{T^{*}}$ & $\frac{h_{\mathrm{A}}^{* \text { mech }}-\sqrt{m_{\mathrm{A}} / m_{\mathrm{B}}} h_{\mathrm{B}}^{* \text { mech }}}{T^{*}}$ & $\frac{h_{\mathrm{A}}^{*}-\sqrt{m_{\mathrm{A}} / m_{\mathrm{B}}} h_{\mathrm{B}}^{*}}{T^{*}}$ \\
\hline A1 & -0.548 & -4.398 & 1.057 & -5.999 & 3.850 & 7.056 \\
$\mathrm{~A} 3$ & -0.775 & -4.048 & 0.620 & -5.440 & 3.273 & 6.060 \\
$\mathrm{~A} 5$ & -0.986 & -3.876 & 0.366 & -5.224 & 2.890 & 5.590 \\
B1 & -1.727 & -3.401 & -3.675 & -1.451 & 1.674 & -2.224 \\
$\mathrm{~B} 3$ & -1.658 & -2.919 & -3.008 & -1.468 & 1.261 & -1.540 \\
$\mathrm{~B} 5$ & -1.858 & -2.725 & -3.244 & -1.332 & 0.867 & 1.386 \\
$\mathrm{Ar}-\mathrm{Kr}$ & -2.737 & -5.667 & -2.619 & -5.800 & 1.176 & \\
\hline
\end{tabular}

portionally to the mole fractions. These latter enthalpy values have been used to compute the transport coefficients presented in [6]. We shall denote these approximate values by $h_{\alpha}^{\text {mech }}$.

Evidently the values of $\Delta h$ deviate substantially from the approximate ones-even for systems A3 and B3, which are the most ideal mixtures in the sense that the potential parameters for unlike interactions represent the means of the parameters for like interactions.

These significant discrepancies lead us to expect that the thermal conductivity, $\lambda$, and the thermal diffusion coefficient, $D^{\mathrm{T}}$, computed in [6] on the basis of values of $h_{\alpha}^{\text {mech }}$ have to be corrected considerably. Fortunately the effect of the enthalpy term on the autocorrelation function of the heat current is negligibly small, as shown in [9]. Thus $\lambda$ is not influenced by the incorrect determination of $h_{\alpha}$. However, the thermal diffusion coefficient is markedly dependent on the values of $h_{\alpha}$, as we have demonstrated in terms

Table 5. Thermal diffusion coefficients $\dagger$ and their partial contributions ( from the DP. DE and DK parts of the Green-Kubo integral [6]) for the mixture systems considered in table 1, and $\mathrm{Ar}-\mathrm{Kr}$.

\begin{tabular}{lcrllrl}
\hline System $\ddagger$ & $D_{\mathrm{DP}}^{\mathrm{T}}$ & $D_{\mathrm{DE}}^{\mathrm{T}}$ & $D_{\mathrm{DE}}^{\mathrm{T}, \text { mech }} \S$ & $D_{\mathrm{DK}}^{\mathrm{T}}$ & $D^{\mathrm{T}}$ & $D^{\mathrm{T}, \mathrm{mech}}$ \\
\hline $\mathrm{A} 1$ & 5.45 & -8.89 & -4.85 & - & -3.44 & 0.60 \\
$\mathrm{~A} 3$ & 3.83 & -6.08 & -3.28 & - & -2.25 & 0.55 \\
$\mathrm{~A} 5$ & 3.31 & -5.15 & -2.66 & - & -1.84 & 0.65 \\
$\mathrm{~B} 1$ & 0.17 & 1.95 & -1.47 & - & 2.12 & -1.30 \\
$\mathrm{~B} 3$ & 0.08 & 1.81 & -1.63 & - & 1.89 & -1.40 \\
$\mathrm{~B} 5$ & 0.13 & 3.59 & -1.63 & - & 3.72 & -1.50 \\
$\mathrm{Ar}-\mathrm{Kr}$ & -0.302 & -0.684 & -0.580 & 0.452 & -0.53 & -0.43 \\
\hline
\end{tabular}

$\dagger$ In units of $\rho \times 10^{-5} \mathrm{~cm}^{2} \mathrm{~s}^{-1}$, where $\rho$ denotes the mass density of the mixture. The statistical error for $D^{\mathrm{T}}$ is about $20 \%$ (see [3] and [6]).

$\ddagger$ Equi-molar composition.

$\S$ Approximate value based on $h_{\alpha}^{\text {mech }}$ (see table 4). 
of the three partial cross correlation functions (CCFs) constituting the total one [3]. We have the diffusive potential (DP), the diffusive enthalpic (DE) and the diffusive kinetic (DK) part. Each term contributes to the total CCF in the same order of magnitude. A detailed calculation shows that the DE term is directly proportional to $h_{\mathrm{A}}-$ $\left(m_{\mathrm{A}} / m_{\mathrm{B}}\right)^{1 / 2} h_{\mathrm{B}}$, where $m_{\mathrm{A}}$ and $m_{\mathrm{B}}$ denote the masses of the component particles [10].

The partial contributions to the coefficient $D^{\mathrm{T}}$ have not been given in [6], and we present them here together with the corrected values, evaluated on the basis of the 'exact' values of $h_{\alpha}$, and the total transport coefficients, in table 5. Our choice of equal masses of the particles of the mixtures of types $\mathrm{A}$ and $\mathrm{B}$ ensures that there is no DK part present. Consequently the DE part is about half of the total $D^{\mathrm{T}}$, and $\Delta h$ computed by the DM can be used directly, as the mass factor is unity.

Table 5 shows the appreciable changes that result for the coefficient $D^{\mathrm{T}}$ of the various mixtures. Although even the sign of the transport coefficient is reversed, the trends found in [6] remain approximately preserved.

In particular, we have opposite signs for the transport coefficients of systems of type A and type B. Noticing the large error bars of these coefficients, we see that there is only a slight dependence on the particular system of a given type. The sign change of $D^{\mathrm{T}}$ on going from type A to type B occurs for the following reasons: as isotopic systems are considered, no DK contribution to the total correlation function and hence the total $D^{\mathrm{T}}$ exists. Thus the transport coefficient is composed of DP and DE terms only. For these non-ideal mixtures the thermodynamic contribution, i.e. the DE term, gives the dominant part of $D^{\mathrm{T}}$. It is known that thermodynamic properties of $\mathrm{LJ}$ mixtures are oppositely affected when the $\sigma$-parameter of a chosen particle species is altered instead of $\varepsilon$ [11].

\section{Discussion and conclusions}

We have shown that the DM works just as well for non-ideal stable mixtures of the LJ type as for the more ideal mixtures studied in [4]. It gives partial enthalpies accurate to within $2-3 \%$, at least for systems of type A. One useful indication of the suitability of the method for determining thermodynamic properties is the good agreement of results obtained by virtually mutating a particle of species $\mathrm{A}$ into species B and a particle of species B into species A.

As expected, the $\Delta h$ values obtained using this method differ appreciably from those computed by 'sharing' the total enthalpy among the components. Accordingly, the thermal diffusion coefficient determined using the approximated values of $h_{\alpha}$ is not correct for the model mixtures considered, A1, A3, A5 and B1, B3, B5. In [6] we have, however, anticipated this.

Surprisingly, significant corrections for $D^{\mathrm{T}}$ are also necessary for the LJ Ar-Kr mixture because of the large $m_{\mathrm{Ar}} / m_{\mathrm{Kr}}$ ratio. The partial contributions to $D^{\mathrm{T}}$ and the total value for $\mathrm{Ar}-\mathrm{Kr}$ are given at the bottom of table 5. Although $h_{\mathrm{A}}-h_{\mathrm{B}}$ exceeds $h_{\mathrm{A}}^{\text {mech }}-h_{\mathrm{B}}^{\text {mech }}$ by only $10 \%$ (see table 4 ), the weighting factor $\left(m_{\mathrm{Ar}} / m_{\mathrm{Kr}}\right)^{1 / 2}$ leads to a final enlargement of the absolute value of $D^{\mathrm{T}}$ by $25 \%$.

Two concluding remarks might be in order. Using the approximate values of $h_{\alpha}$ for the determination of $D^{\mathrm{T}}$, i.e. the cross correlation function, the latter always starts at the origin. However, when the correct values of $h_{\alpha}$ are used-for instance, as determined using the DM-the CCF commonly has a non-zero initial value.

In equilibrium MD computations, one may easily include the 'exact' thermodynamic values of $h_{\alpha}$ to determine the correct transport coefficient. By contrast, in non-equi- 
librium MD, the incorporation of these values of $h_{\alpha}$ is not obvious, and a suitable technique for doing this has not been proposed as yet [12].

\section{Acknowledgments}

We thank the 'Rechenzentrum der Ruhr-Universität Bochum' for a generous amount of computer time on the Cyber 205 vector machine and U Krupinski for typing as well as B Weinert for graphics. We are particularly indebted to the director of CECAM, Carl Moser, for his kind hospitality during a workshop in 1987.

\section{References}

[1] Bearman R J 1958 J. Chem. Phys. 291278

[2] Gillan M J 1987 J. Phys. C: Solid State Phys. 20521

[3] Vogelsang R, Hoheisel C, Paolini G V and Ciccotti G 1987 Phys. Rev. A 363964

[4] Sindzingre P, Ciccotti G, Massobrio C and Frenkel D 1987 Chem. Phys. Lett. 13635

[5] Sindzingre P, Massobrio C, Ciccotti G and Frenkel D 1988 Chem. Phys. at press

[6] Vogelsang R and Hoheisel C 1988 Phys. Rev. A at press

[7] McDonald I R 1973 Statistical Mechanics vol 1, ed. K Singer (London: The Chemical Society)

[8] Torrie G M and Valleau J P 1977 J. Chem. Phys. 661402

Schoen M and Hoheisel C 1984 Mol. Phys. 531367

[9] Vogelsang R and Hoheisel C 1987 Phys. Rev. A 353487

[10] Hoheisel C and Vogelsang R 1988 Comp. Phys. Rep. at press

[11] Schoen M and Hoheisel C 1986 Mol. Phys. 57 65; Hoheisel C 1988 Mol. Phys. at press

[12] Paolini G V and Ciccotti G 1987 Phys. Rev. A 355156 